\title{
Ein multifunktionales Herzkranzgefäßmodell: Nutzungsvarianten für vergleichende Untersuchungen an PTCA-Kathetern
}

\author{
Kraft M., Rutsch W.*, Boenick U. \\ Institut für Mikrotechnik und Medizintechnik, Technische Universität Berlin \\ * Zentrum für Innere Medizin, Medizinische Klinik und Poliklinik I, Charité, Humboldt Universität Berlin
}

\section{EINLEITUNG}

Obwohl neue koronarinterventionelle Techniken ihre Eignung für die minimal invasive Therapie der koronaren Herzkrankheit unter speziellen Indikationsstellungen nachweisen konnten, ist die Ballondilatation (PTCA) bis heute das Standardverfahren zur Revaskularisierung verengter Koronargefäße. Das Verfahren belegt damit eine Mittelstellung zwischen medikamentöser Therapie und Bypassoperation. Der Erfolg der Operationstechnik liegt neben ihrer Einfachheit und des vergleichsweise geringen Bedarfs an Zubehör im wesentlichen darin begründet, da $B$ allein bei der Ballondilatation kein die Flexibilität des Katheters einschränkender Zusammenhang zwischen Durchmesser des Anwendungsteiles und dem erzielbaren freien Lumen im Gefäß besteht. Die Kombinationsmöglichkeit der konventionellen PTCA mit der Applikation von Gefäßendoprothesen (Stents) sichert dem Verfahren eine zusätzliche Erweiterung seiner Anwendbarkeit.

\section{UNTERSUCHUNG VON PTCA-KATHETERN}

Derzeit sind eine Vielzahl von PTCA-Kathetern verschiedener Hersteller verfügbar. In Deutschland haben sich "Monorail" bzw. "Rapid-Exchange"-Katheter nach Bonzel durchgesetzt, bei denen der Führungsdraht nur durch den distalen Teil des Katheters läuft. Alle Katheter unterscheiden sich, zum Teil gravierend, in ihren Eigenschaften. Dies liegt auch darin begründet, daß eine angestrebte Merkmalsausprägung nur über Kompromisse erreichbar ist. So erfordert z.B. ein hoher Ballonberstdruck in der Regel eine geringfügig größere Ballonwandstärke, welche mit Einschränkungen in der Flexibilität des Katheters verbunden ist.

Ein am Institut für Medizintechnik entwickelter multifunktionaler Prüfstand erlaubt die Untersuchung der Eignung eines Katheters für verschiedene Indikationsstellungen.

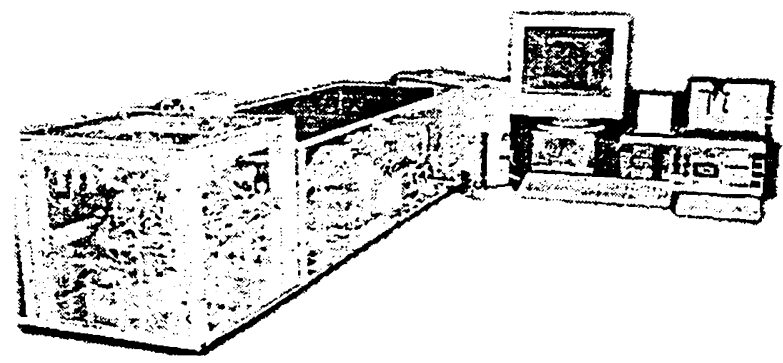

\section{Abb. 1: Prüfstand für PTCA-Katheter}

Das integrierte Modell des Koronargefäßsystems ist in Geometrie und der Nachbildung der Reibverhältnisse den physiologischen Gegebenheiten nachempfunden. PTCAKatheter werden bei einer Temperierung des Gefäßmodells auf $37^{\circ} \mathrm{C}$ in Wasser untersucht, wobei neben
Vorschubkräften verschiedene Reaktionskräfte im Gefäßsystem sowie Drücke im Ballon und in Modellstenosen meßbar sind.

\section{LAGE DER SENSOREN}

Konzept [1] und Aufbau [2] der Modellsensorik wurden bereits vorgestellt. Der Schwerpunkt dieses Beitrages liegt nun nach Fertigstellung des Prüfstandes auf der Beschreibung der Sensorpositionen und der möglichen Messungen im Modell. Erste Meßergebnisse enthalten die die Beiträge [3,4].

Die Plazierung der Sensoren im Modell erfolgte unter Berücksichtigung der Forderungen:

- Gewährleistung einer, alle wesentlichen Kathetereigenschaften beinhaltenden Untersuchung, d.h. die erfaßten Meßwerte sollen als Datenbasis für eine indikationsspezifische Katheterbewertung [5] verwendbar sein,

- Realisierung einer realitätsnahen Meßwerterfassung, d.h. Katheterkennwerte werden an den anatomischen Positionen untersucht, wo sie während der Intervention eine Rolle spielen.

Im Koronargefäßmodell sind fünf Reaktionskraftaufnehmer positioniert, die in beliebiger Kombination, in der Regel jedoch gemeinsam mit einer Messung der notwendigen Verschubkräfte am Antrieb (Aktionskraft), genutzt werden können. Messungen des Ballon- und Reaktionsdruckes bei Balloninflation in Modellen von Verengungen (Stenosen) sind ebenfalls möglich. Eine zusätzliche Laser-Durchmesser-Prüfvorrichtung dient der zweiachsigen Vermessung der Katheterballons in Abhängigkeit vom erreichten Innendruck [4].

Eine Darstellung der Sensorpositionen im Modell, orientiert an der segmentalen Einteilung nach "American Heart Association", enthält die Abb. 2. Nachfolgend werden die dort bezeichneten Sensoren erläutert:
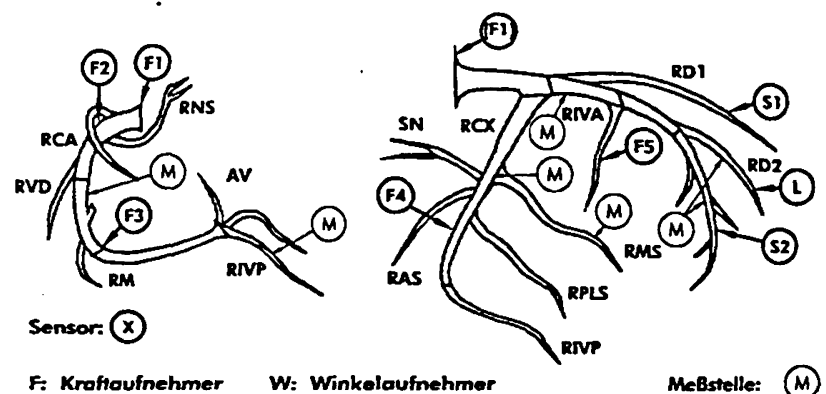

F: Krofioufnehmer S: Stenosenmodell
L: Lichtschranke W: Winkolaufnehmer externe Sensoren: Mebstelle: (M)

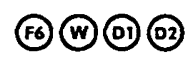

\section{Abb. 2: Lage der Sensoren im Modell}

S1) Das Modell einer Verengung (Stenose) im RD1 eignet sich zur Simulation exzentrischer Stenosen mit Längen zwischen 4 und $32 \mathrm{~mm}$. Am Umfang eines zentral 
liegenden Gefäßmodells sind neun einzeln befüllbare Druckschläuche angeordnet, deren äußere Abstützung in wechselbaren Blenden (Einstellung der Länge) erfolgt.

S2) Im distalen RIVA befindet sich ein Stenosenmodell, welches aus einer volumenkonstanten Kammer mit einem integrierten Gefäßsegment besteht [2]. In dem Modell sind über eine Druckbeaufschlagung des Gefäßsegmentes verschiedene Stenosegrade einstellbar. Zusätzlich können Kammerdrücke bei Inflation des Ballons erfaßt und einer Ballonaufweitung entgegenwirkende Kräfte (entsprechend der physiologischen Beanspruchung in festen Stenosen) erzeugt werden.

F1) Ein Kraftaufnehmer am Ostium des RCA dient der Messung von Abstützkräften des PTCA-Katheters im Führungskatheter (Backup). Während der OP können zu hohe Abstützkräfte zu einer Deplazierung des Führungskatheters (Verlagerung aus dem Ostium) und damit zu einem Abbruch der Intervention führen.

F2/F3) Zwei Kraftaufnehmer in Gefäßkrümmungen (medialer RCA und RVD) sind geeignet, radial auf die Gefäßwand wirkende Kräfte bei Passage eines Katheters oder Aufrichtkräfte bei Dilatation eines Ballons in der Gefäßkrümmung zu messen [2,3].

F4) Ein im Gefäßverlauf befindlicher Kraftaufnehmer (medialer RCX) verfügt am Krafteinleitungspunkt über wechselbare Blenden. Mit ihm können Reaktionskräfte am Führungsdraht, durch den Katheter applizierbare Kräfte und Stent-Abstreifkräfte gemessen werden [3].

F5) Ein weiterer Kraftaufnehmer (Septalast des RIVA) dient vergleichenden Untersuchungen des Ballonaufweitverhaltens. Er ist über einem Silikon Gefäßsegment mit Messinghülse angeordnet, welche ausschließlich unter dem Kraftaufnehmer gefenstert ist. Simuliert wird eine stark kalzifizierte Stenose mit auftretender Dissektion.

L) Ein Sensor mit integrierter Lichtschranke im RD2 ist für die Untersuchung der radialen Reibung des Führungsdrahtes im PTCA-Katheter geeignet [2]. Dieser spannt sich bei proximaler Drehung am Antrieb zunächst wie eine Torsionsfeder und entlädt sich im Anschluß durch eine distale Drehung. Dabei wird eine die Lichtschranke unterbrechende Blende kurzzeitig geöffnet und die Drehwinkelmessung am Antrieb gestoppt.

M) Weitere Meßstellen im Modell besitzen keine eigenen Sensoren. Beisiele für solche Gefäßabschnitte sind:

a) Ein Abschnitt im medialen RCA, welcher für Wandreaktionskraftmessungen (F2) nutzbar ist, kann im wechselbaren Gefäßabschnitt auch für den Einsatz eines Stenosenmodells verwendet werden. Derartige "feste" Stenosen liefern im Vergleich zu den bereits beschriebenen einstellbaren Stenosen (S1, S2) eine bessere Reproduzierbarkeit der meßbaren Vorschub- und Abstützkräfte im Führungskatheter.

b) Das Modell einer plastisch verformbaren Stenose (Schaumstoff) befindet sich im RMS. Hicr erreichte Ballongeometrien können nach Wirkung einer für die Verformung der Stenose notwendigen und der Ballonentfaltung entgegenwirkenden Kraft untersucht werden.

c) Distal, 2.B. im RIVP liegende Meßstrecken, eignen sich zur Bestimmung maximal möglicher Eindringtiefen des Katheters. Das Ziel der Messungen besteht hier in der Aufzeichnung notwendiger Vorschubkräfte.

\section{NUTZUNGSVARIANTEN}

Die möglichen Nutzungsvarianten des Prüfstandes ergeben sich aus den bereits erläuterten Zweckbestimmungen der Sensoren und ihrer Anordnung im Modell, d.h. ihrer Kombinationsfähigkeit. Einige typische Messungen sind:

- Messung von Vorschubkräften an Stenosen verschiedener Längen, Stenosegrade und radialer Geometrien (medialer RCA, RD1: S1, RIVA: S2) vor und nach Balloninflation zur Untersuchung der Crossability bzw. des Recrossingverhaltens,

- Messung des Ballondruckes und des Reaktionsdruckes in einem volumenkonstanten Stenosenmodell (RIVA: S2),

- Messung des Balloninnendruckes und der Aufrichtkraft in einem gekrümmten Gefäßsegment mit und ohne Stent (medialer RCA: F2),

- Messung des Balloninnendruckes und der Aufweitkraft in einem gefensterten Modellgefäßsegment (RIVA: F5),

- Messung von Vorschub- bzw. Rückzugkraft im RCA in Kombination mit einer Messung der Wandreaktionskräfte (F2) und Abstützkräfte (F1) im Führungskatheter [3],

- Messung von Vorschubkräften im RCX in Kombination mit Reaktionskräften am Führungsdraht oder Abstreifkräften an einem Stent (F4) bzw. Messung der Kraftdifferenz $\mathrm{F}_{\text {Antrieb }}-\mathrm{F}_{\mathrm{Gefa} B}$ am gleichen Aufnehmer,

- Messung des notwendigen proximalen Drehwinkels am Führungsdraht bis zum erstmaligen Auftreten einer distalen Drehung (RD2: L),

- Externe Messung des Ballondurchmessers in Abhängigkeit vom Balloninnendruck [4].

Das Gefäßmodell ist an andere Messungen anpaßbar. Der zur Zeit am RCA positionierte Aufnehmer der Abstützkraft im Führungskatheter (F1) kann auch am MLCA positioniert werden. Weiterhin lassen wechselbare Gefäßsegmente (RCA) die Verwendung beliebiger Einsätze zu. So sind z. B. Messungen des Ballonberstdruckes an Stenosenmodellen mit scharfkantigen Kalkeinlagerungen vorgesehen.

\section{LITERATUR}

[1] Kraft, M.; Boenick, U.: Konzeption eines anatomischen Herzkranzgefäßmodells für vergleichende Untersuchungen an PTCA-Kathetern, Zeitschrift Biomed. Tech-nik, Band 41 (Erg.band), 1996, S. 472-473

[2] Kraft, M.; Wendt, O.; Rutsch, W.; Boenick, U.: Realisierung eines Katheterantriebes und der MeBwerterfassung in einem Herzkranzgefäßmodell für PTCAKatheter, Zcitschrift für Biomed. Technik, Band 42 (Erg.band), 1997, S. 484-485

[3] Kraft, M.; Rutsch, W.; Boenick, U.: Reaktionskraftuntersuchungen bei Vorschub und Rückzug von PTCAKathetern in modellierten Koronargefäßen, Zeitschrift für Biomed. Technik, Band 43 (Erg.band), 1998

[4] Kraft, M.; Rutsch, W.; Boenick, U.: Untersuchung der zeitabhängigen Ballonaufweitung an PTCA-Kathetern, Zeitschrift für Biomed. Technik, Band 43 (Erg.band). 1998

[5] Kraft, M.; Marsolck, 1.; Rutsch, W.; Bocnick U.: Ein Fuzzy-Bewertungsprogramm für PTCA-Katheter. Zeitschrift für Biomed. Technik, Band 42 (Erg.tand), 1997, S. 439-442 